\title{
MULTI-FUNCTIONAL INTERFACE FOR FLEXIBILITY AND RECONFIGURABILITY OF FUTURE EUROPEAN SPACE ROBOTIC SYSTEMS
}

\author{
Javier Vinals $^{\text {a* }}$, Eduardo Urgoitia ${ }^{a}$, Gonzalo Guerra ${ }^{a}$, Ignacio Valiente ${ }^{b}$, Judit Esnoz-Larraya ${ }^{\text {b }}$, Michel \\ Ilzkovitz ${ }^{\mathrm{c}}$, Mario Franćeskic ${ }^{\mathrm{c}}$, Pierre Letier ${ }^{\mathrm{c}}$, Xiu-Tian Yan ${ }^{\mathrm{d} *}$, Gwenole Henry ${ }^{\mathrm{d}}$, Albino Quaranta ${ }^{\mathrm{e}}$, Wiebke \\ Brinkmannf, Marko Jankovic ${ }^{\mathrm{f}}$, Sebastian Bartsch ${ }^{\mathrm{f}}$, Alessandro Fumagalli ${ }^{\mathrm{g}}$ Manfred Doermer $^{\mathrm{h}}$
}

\author{
a SENER Ingenieria y Sistemas, S.A., Spain, javier.vinals@ sener.es, eduardo.urgoiti@sener.es, \\ gonzalo.guerra@sener.es \\ bMag Soar, Spain, ivaliente@magsoar.com, jesnoz@magsoar.com \\ c Space Applications Services N.V., Belgium, michel.ilzkovitz@ spaceapplications.com, \\ mario.franceski@spaceapplications.com,pierre.letier@spaceapplications.com \\ ${ }^{\mathrm{d}}$ University of Strathclyde, United Kingdom, x.yan@strath.ac.uk, gwenole.henry@strath.ac.uk \\ e Thales Alenia Space, Italy, albino.quaranta@thalesaleniaspace.com \\ ${ }^{\mathrm{f}}$ German Research Center for Artificial Intelligence (DFKI), Robotics Innovation Center, Bremen, Germany, \\ wiebke.brinkmann@dfki.de, marko.jankovic@dfki.de, sebastian.bartsch@dfki.de \\ ${ }^{g}$ Leonardo Spa, Italy, alessandro.fumagalli@leonardocompany.com \\ ${ }^{h}$ Airbus Defence and Space GmbH, Germany, manfred.doermer@airbus.com \\ *Corresponding Authors: javier.vinals@sener.es, x.yan@strath.ac.uk
}

\begin{abstract}
The capabilities of maximising standard payload modules' functionalities for applications such as on-orbit satellite servicing or planetary exploration depend critically on the creation and availability of a standard interface (IF). Standard interface should provide, aside from the necessary mechanical interconnections, electrical power and data connections, as well as thermal transfer between "building block" payload modules. The overall flexibility enabled by such IF will allow endless reconfigurations of payload and other modules for different functional requirements. This can be considered a game changer technology, enabling transformation from the current approach to space missions, deploying single-use system with pre-planned and limited functionalities, to a radically new approach with multi-use, dynamically reconfigurable and multi-functional systems. Hence, SIROM aims to set a new research agenda for future affordable space missions.

Within this context, the partners of the SIROM (Standard Interface for Robotic Manipulation of payloads in future space missions) project are developing the first standard IF solution that combines the four required functionalities in an integrated and compact form for future space missions. With a mass lower than $1.5 \mathrm{~kg}$ and having an external diameter of $120 \mathrm{~mm}$ and a height of $30 \mathrm{~mm}$, this novel interface permits not only mechanical coupling but also electrical, data and thermal connectivity between so called Active Payload Modules (APMs), as well as other modules such as the robotic end-effectors. This multi-functional IF features an androgynous design to allow for replacement and reconfiguration of the individual modules in any combination desired. It consists of the following sub-assemblies: mechanical IF, electrical IF, data IF, thermal IF and IF controller. A clear advantage of SIROM design is that its mechanical IF consists of a latching and guiding systems for misalignment correction, capable of withstanding certain robotic arm positioning inaccuracies: $+/-5 \mathrm{~mm}$ translation and $+/-1.5^{\circ}$ rotation in all axes. Regarding the electrical and data IFs, SIROM transfers up to $150 \mathrm{~W}$ electrical power and provides a data transfer rate of $100 \mathrm{Mbit} / \mathrm{s}$ via SpaceWire, plus command communication with speeds up to $1 \mathrm{Mbit} / \mathrm{s}$ via CAN bus. The thermal IF provides fluidic ports for flow transfer and has the potential to transfer 2500W between APMs accordingly provided with the corresponding closeloop heat exchange system. Although not envisaged for SIROM current design, a possible variation could be to use these ports for satellite re-fuelling. Apart from that, SIROM exhibits redundant coupling capabilities: it can match and couple another completely passive SIROM. It is provided with main and redundant connectors for thermal, electrical, data and control flow in case of one of the lines fails. All in all, SIROM will enable long duration missions with no logistic support, refurbishing, maintenance and reconfiguration of satellites, cost efficiency and simplification of the tool exchange in scientific exploration missions. SIROM is designed to be a common building block for European and possibly world future space robotics enabled missions.
\end{abstract}

Keywords: space robotic, space interface, orbital missions, orbital payload, standard interface, multifunctional interface

Page 1 of 14 


\section{Acronyms/Abbreviations}

Active Payload Module (APM), Interface (IF), Standard Interface for Robotic Manipulation of Payloads in Future Space Missions (SIROM), Electrical Ground Support Equipment (EGSE), On-Orbit Servicing (OOS), low Earth orbit (LEO), multi-robot system (MRS), On Board Computer (OBC).

\section{Introduction}

A full connectivity among many functional payload modules enables a new cost-effective and flexible way of designing future space missions. This connectivity will create a new space mission design paradigm in which a plug-play space system can be designed for planetary exploration or on-orbit servicing. It is envisaged these new systems will generate significant benefits in cost reduction, functionality expansion and many other quality improvements which otherwise would not be possible.

The Standard Interface for Robotic Manipulation of Payloads in future space missions (SIROM) is a project funded by the EU Horizon 2020 research and innovation program aiming to advance significantly the European's space robotics capabilities and realise the required connectivity, by developing a standard interface for onorbit and planetary missions. This is achieved by realising the connectivity among various payload and other modules in the form of mechanical, data, electrical and thermal/flow connections in a compact and integrated form. The SIROM project [1] [2] includes the design and development of the standard interface and its controller, as well as a set of orbital and planetary active payload modules (APMs), a robot arm end-effector, and the EGSE as well as the verification tests to be performed. Specifically SIROM interface is designed to provide mechanical, data, electrical and thermal/flow connectivity in a compact and integrated form. The developed standard interface allows the coupling of an active payload module (APM) to a robot manipulator, APM to other APM and APM to a platform. SIROM interfaces hence enables constructing a modular, scalable and reconfigurable systems, based on the interconnection of APMs and a spacecraft structure.

This paper includes sections on the state of the art, SIROM design requirements and constraints, reference mission scenarios, overview of the SIROM system and description of the system design, results of simulations and tests and future applications, ending with discussion and conclusions.

\section{The state of the art}

Today's spacecraft both for orbital or planetary missions is unique designed to one specific mission. No adaptations to changing needs on long duration missions nor repair of failed systems is foreseen. Future spacecrafts due to the changing business perspectives and needs will request more versatile and adaptable solutions. Together with the progress in autonomous space robotics (e.g. RESTORE-L, DEOS) the SIROM projects provides an enabler to this approach by:

1. developing an interface which is compatible to robotic manipulation and provides a standardized modular transfer of mechanical, data, power and thermal loads.

2. developing standardized payload containers (APM's) which enhances the overall mission flexibility. The APM allows the reconfiguration, upgrading or repair of spacecrafts by combining the SIROM interfaces with integrated payloads.

The design requirements for the SIROM interface define a versatile, scalable and modular lay-out which supports standardization of the mechanical interface, the data, thermal and power modules. Together with the active payload module (APM) this system constitutes a building block to allow the design of modular, scalable and reconfigurable space systems. An autonomous servicer spacecraft equipped with a manipulator can reconfigure, repair or assemble satellites in orbit by installing APMs in space. With the SIROM interface the coupling of APMs to larger assemblies in space is also possible. Future planetary missions consisting of lander and rovers will call for interaction between themselves and additional payload elements on the planetary surface. SIROM APMs and interfaces offer the flexibility required to play a crucial role in future planetary missions.

In addition to the above industrial view, the literature reviews conducted in previous SIROM papers drew up an overview [3] of power, data, thermal and mechanical functionalities in interfaces already used in robotics and space applications. A few devices integrating more than one of these functionalities are also presented in [4]. Presently operating or still at the development stage, only one of these products includes all 4 aforementioned functionalities[5], and is mostly designed for space orbital missions. The prototype developed by SIROM is, on the other hand, designed to be deployed in both orbital and planetary exploration environments.

Space industry is looking for enablers to foster new capabilities and technology-led business opportunities in the space sector. This trend is driven by the new launchers making access to space cheaper and quicker, plus new technologies which shift the technology from single shot entity to a reconfigurable platform. Important stakeholders such as ESA and its member states, NASA, DARPA and EU support this technology development by setting up technology projects or missions, e.g. :

- $\quad$ ASSIST (ESA)

- $\quad$ SUMO (NASA)

- $\quad$ RESTORE-L (NASA) 
- $\quad$ RRM-3 (NASA)

- $\quad$ iBoss (DLR)

- $\operatorname{SIROM}(\mathrm{EU})$.

Other space fairing nations like Russia, China, Japan and India have also defined their roadmaps in the emerging fields of on-orbit servicing and on-orbit assembly. The pioneer project for on-orbit servicing technology is NASA Orbital Express (launched 2007) with:

- $\quad$ First autonomous fuel transfer performed

- First autonomous replacement of an Orbital Replacement Unit (ORU)

- First autonomous replacement of equipment (battery, computer).

There are two basic elements for any reconfigurable space system:

- A standardized payload container (APM)

- A standardized interface between the APMs or the APMs and the spacecraft.

- Docking and undocking of the APMs is performed by a robotic manipulator.

A standard interface has to provide the following features:

- $\quad$ Transferring mechanical loads

- Transferring power

- Transferring data

- Transferring heat loads (optional)

- Transferring fluids (optional).

Although servicing of space system is common in the context of the international space station (performed by humans), autonomous robotic servicing in space is nascent.

On-Orbit Servicing can be subdivided into:

- Servicing of space system already in space and not designed for servicing (non-cooperative target)

- $\quad$ Servicing of space systems designed to service and be serviced (cooperative target)

- On orbit assembly

Planetary or orbital, future space system architecture will consist of standardized, reconfigurable subsystems (e.g. APM) that can be replaced in case of failure or added to improve, upgrade or provide extra capabilities of the system as a whole. These standardized subsystems could also be structural building blocks, used to assemble large structures in space or on the planet surfaces, by using standard elements with standard interfaces.

\section{SIROM design requirements and constraints}

The requirements for SIROM interface design comes from an extensive literature review, the project call and industrial partner's inputs and were refined by the consortium. These include functional requirements specific to the interface performances. The most challenging functional requirements that have constrained the design are summarized below:

- Capability to withstand $200 \mathrm{~N}$ and $40 \mathrm{Nm}$ combined in the worst-case direction;

- Capability to withstand a sine level of $10 \mathrm{~g}$ up to $100 \mathrm{~Hz}$, and $15 \mathrm{~g}$ static level;

- Capability to transfer $150 \mathrm{~W}$ electrical power whilst providing overcurrent and under-voltage protections;

- Capability to transfer data at a rate of $100 \mathrm{Mbit} / \mathrm{s}$.

- Capability to transfer $2-2,5 \mathrm{~kW}$ thermal load via fluidic port;

- Capability to compensate $5 \mathrm{~mm}$ translational and $1^{\circ}$ rotational position inaccuracies.

In addition, the following environmental requirements are summarized below:

- SIROM shall withstand very demanding thermal, radiation and dust environments;

- Minimum 10000 mating/de-mating cycles;

- Exposure to space environment for 15 years;

- Exposure to Mars environment for 1 Martian year (687 Earth days);

- Capability of surviving $-128^{\circ} \mathrm{C}$ to $+50^{\circ} \mathrm{C}$ temperature range, in both connected or not connected configuration;

- Capability of modules connections operating within a temperature range of $-110^{\circ} \mathrm{C}$ to $+50^{\circ} \mathrm{C}$;

- Robustness and reliability even in presence of very abrasive or magnetic dust.

One of the other main challenges is to minimize the volume needed to integrate all subsystems. This drives the effort of the overall SIROM design and the choice of components and connectors.

\section{Reference mission scenarios}

\subsection{Orbital mission operations scenario}

The orbital reference scenario under which the developed multifunctional interface shall be used, has been envisioned to consist of a robotic on-orbit servicing (OOS) scenario, where the servicer spacecraft performs a servicing task on a cooperative, modular client spacecraft in low Earth orbit (LEO), as illustrated in Fig. 1. In particular, the OOS shall consist of a replacement of a faulty/outdated payload, such as an optical sensor, integrated onto the primary bus of the client spacecraft via an APM. The exchangeable module shall be equipped with at least two standard interfaces, thus allowing quick and easy access to the desired subsystem via a robotic arm mounted on the servicer. The whole operation shall be performed while the client is firmly berthed to the servicer, thus neglecting the free-floating dynamics of the coupled system [6]. 




Fig. 1. Artist's concept of a robotic on-orbit servicing scenario (credit: NASA/Goddard Space Flight Center 2017)

\subsection{Planetary mission operations scenario}

The planetary scenario is based around a mission to search for volatiles at the lunar poles. The mission involves extended rover operations in shaded lunar craters. For example, the Shackleton crater that lies at the southern lunar pole has an interior that is in permanent darkness while crater rim spends $\sim 80-90 \%$ of the time in sunlight. The reference scenario involves a lunar rover navigating to a crater rim and releasing a solar power charging station, the Primary APM (P-APM), onto the surface in the Sun illuminated area. The P-APM is an autonomous payload that, once dropped on the surface, deploys orientable photovoltaic panels towards the Sun and delivers electrical power to charge a number of swappable battery packs. These battery packs are the Auxiliary APMs (A-APMs). The A-APMs are connected to the P-APM by means of SIROMs that provide power, control, data and thermal interfaces between these devices. In this scenario, the rover has to explore a permanently shadowed region of the crater, so cannot use its own solar panels for recharging its batteries when searching for lunar volatiles. Therefore it will have to return to the P-APM each time it needs a recharge to perform a swap of a depleted A-APM with a fully recharged one. Thanks to this, the rover greatly extends its autonomy and can return in the shadowed area of the crater as often as needed.

\section{SIROM system overview}

With a mass lower than $1,5 \mathrm{~kg}$, SIROM is a cylinder with an external diameter of $120 \mathrm{~mm}, 30 \mathrm{~mm}$ of height above and $30 \mathrm{~mm}$ of height inside an APM. Fig. 2 shows SIROM's main parts. Due to the need to be operative in planetary missions, the external housing and dust cover prevent contamination that would interfere with the proper functioning of the interface.

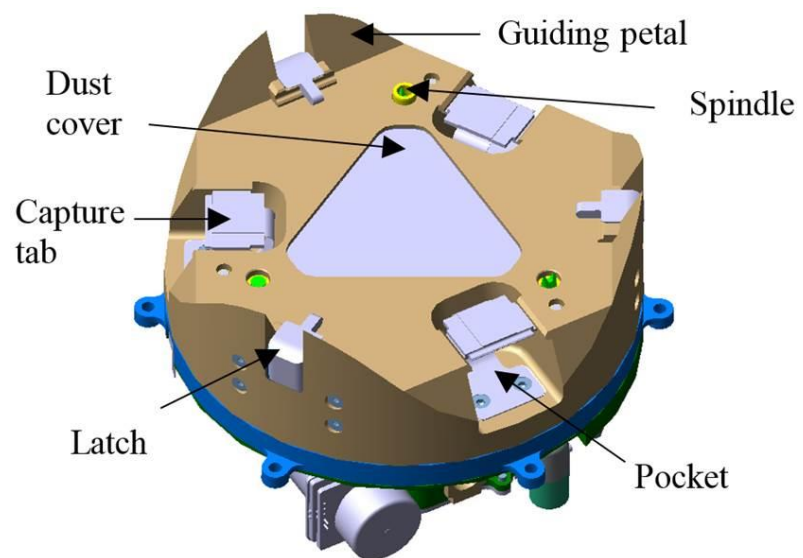

Fig. 2. SIROM interface architecture and main components

SIROMs are directly bolted to an APM structure and in general, the APM could be fitted with multiple SIROMs. Fig. 3 shows the SIROM interface with its APM.

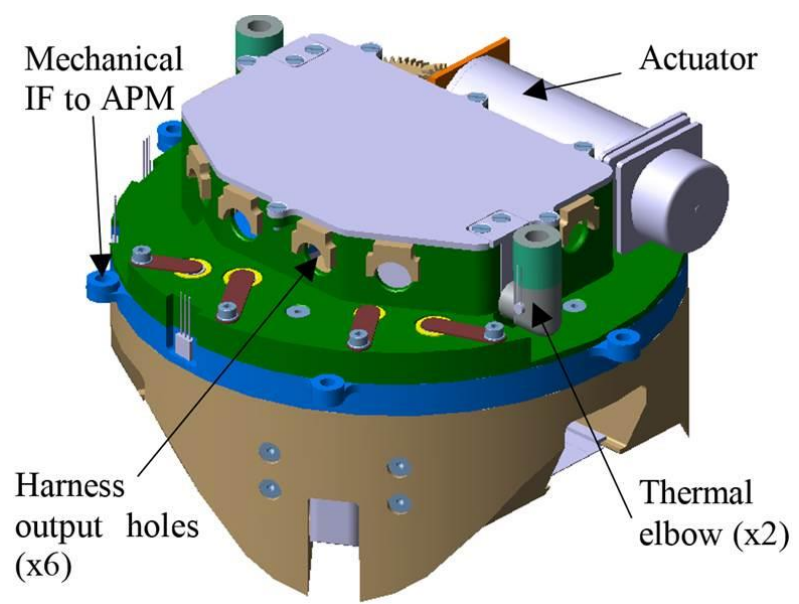

Fig. 3. SIROM interfaces with APM (harness not represented)

SIROM design not only features mechanical, electrical, data and thermal connections in an integrated and androgynous form, but it also presents main and redundant connections in case one of the lines fails. Electrical, data and thermal IFs are located in the socalled connector's plate while the mechanical IF is on its own. Fig. 4 shows the functional interfaces of SIROM at "Connected" position, that is, when the dust cover is opened and the Connectors plate is at the end of its stroke. 


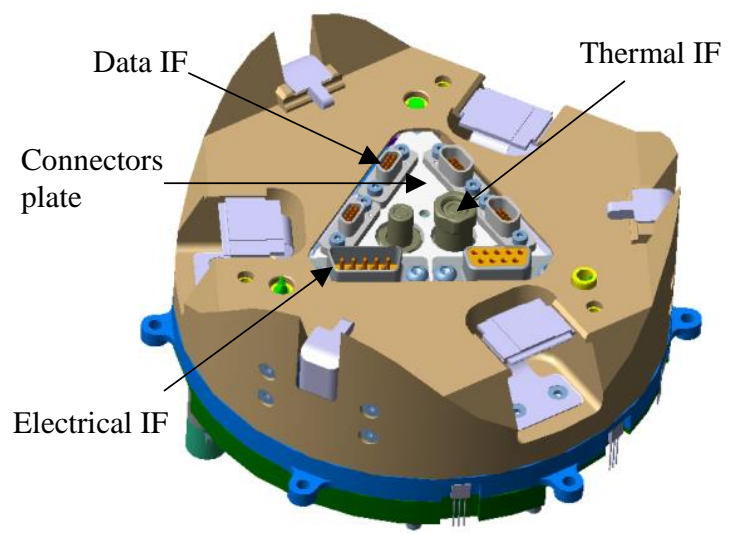

Fig. 4. SIROM functional interfaces

\section{Design approach}

\subsection{Mechanical interface}

The mechanical IF is responsible for providing SIROM berthing capability against another SIROM and also compliance to loads arising during operation, once the attachment is done. This IF is basically formed by three capture hooks (or latches) evenly distributed with $120^{\circ}$ apart, that enter inside the opposite SIROM pockets and retracts. The latches retraction preloads the opposite SIROM capture tabs, resulting in the approximation and compression approach of both SIROMs. Additionally, misalignment errors are self-corrected by the guiding petals during the mentioned approach.

Each latch consists of a titanium four-bar-linkage moved by its own pinion and synchronised by an internal gear. There are three main positions to be distinguished as shown in Fig. 5:

- "Ready to capture": the latches are wide open.

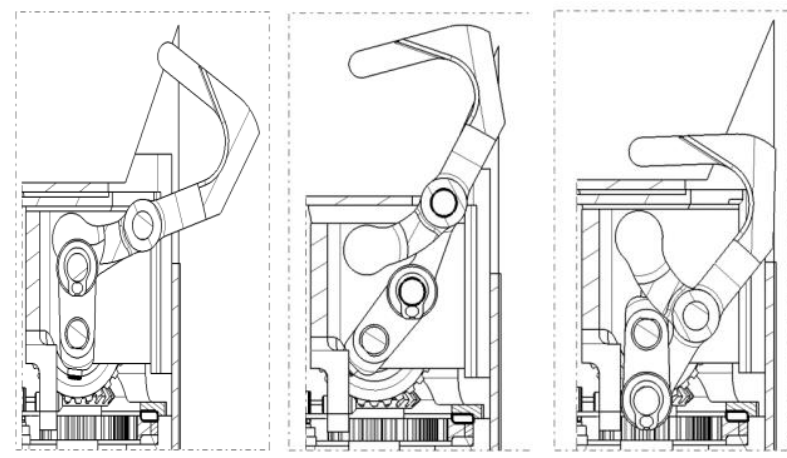

Fig. 5. Latches at "Ready to capture" (left), "Captured" (centre) and "Latched" (right)

- "Captured": the latches are inside the opposite pockets.

- "Latched": the latches are in a locked and secured position; and actuation is required to unlock the latches.

The idea is that a robot manipulator with a SIROM mounted at the end-effector moves to a position in front of the SIROM to be coupled. Then the active hooks go to "Ready to capture" and "Captured" position. From thereon, the latches retraction pulls the opposite SIROM as the preload increases progressively until the latches reach their over-center position. The latches continue the movement a few degrees until they reach an end-stop, corresponding to the "Latched" position. Here the latches are in a blocked and secured position providing a total nominal preload of $1020 \mathrm{~N}$ (i.e. $340 \mathrm{~N}$ per latch).

A clear advantage of the SIROM design is that it is capable of withstanding significant robotic arm positioning inaccuracies. The mechanical IF provides capability to mate an opposite SIROM placed with the following worst combination errors: $10 \mathrm{~mm}$ in axial direction, $5 \mathrm{~mm}$ in other axes and $1,5^{\circ}$ of rotation under all axes.

\subsection{Electrical Interface}

SIROM features an Electrical (power) Interface Subsystem (EIS), which is capable of transferring electrical power in both directions and supplying the SIROM controller. Two lines are provided: one at $100 \mathrm{Vdc}$ and one at $24 \mathrm{Vdc}$. In total, a maximum of $150 \mathrm{~W}$ can be managed by the interface, $120 \mathrm{~W}$ on the $100 \mathrm{~V}$ line and $30 \mathrm{~W}$ on the $24 \mathrm{~V}$ line, which corresponds to 1.2 $\mathrm{A}$ and $1.25 \mathrm{~A}$ respectively. In addition, overcurrent and undervoltage protections are implemented and are automatically triggered. Two supply lines are provided to the SIROM controller: one $5 \mathrm{~V}$ and one at $24 \mathrm{~V}$ not protected.

The interface has a switch, located on the return line, which can be opened or closed by a single logical ON/OFF command. Two signal statuses - one for each line - are used to monitor the presence of power in both lines, in order to connect safely and disconnect the payloads. For safety reasons, no connect or disconnect operation is to be executed without interrupting the power transfer.

The electrical interface consists of a small board (120x120mm); the mass is less than 300 grams and the power dissipated by it is $4 \mathrm{~W}$ in worst scenarios.

The board has wires soldered directly to it, instead of turrets or screw terminals, in order to reduce overall thickness. The SIROM external connectors are D-sub 9pin. In order to have a more versatile hardware during testing of all possible scenarios, all connections between EIS and SIROM, shown in Fig. 6, are made with intermediate connectors to reduce the volume as much as possible.

Small connectors have been selected (i.e. of Molex type). 


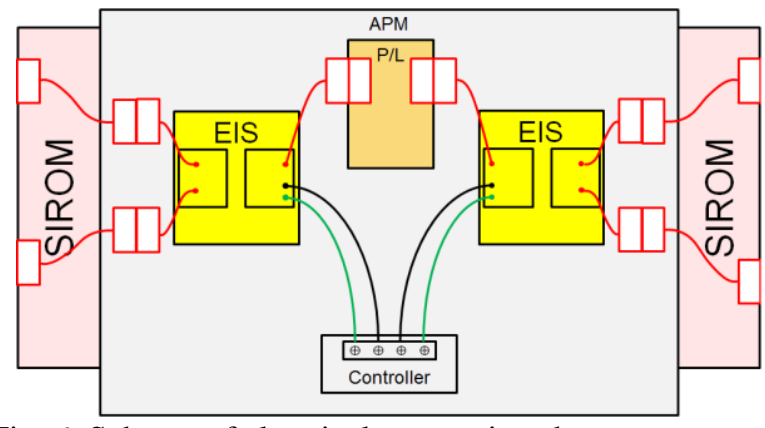

Fig. 6. Scheme of electrical connections between two EIS and two SIROM.

\subsection{Thermal Interface}

The objective of the thermal IF is to provide a high heat exchange, up to $2500 \mathrm{~W}$, between two APMs. The thermal IF shall be able to operate in a wide temperature range $\left(\left[-40\right.\right.$ to $\left.50^{\circ} \mathrm{C}\right]$ for the orbital scenario and $[-110$ to $\left.50^{\circ} \mathrm{C}\right]$ for the planetary scenarios). The maximum temperature jump between SIROM connected IFs operating at maximum power conditions shall be less than $10^{\circ} \mathrm{C}$ and be able to operate for a relatively large number of mating and de-mating cycles $>1000$ (w.r.t. Req of 10000 cycles as a minimum), minimizing the fluid leakage during the process. Due to commercial fluidic components used in the demonstrator, Thermal I/F shall be able to operate for a minimum of 1000 mating and demating cycles. The mating/demating cycling shall also minimize the fluid leakage during the process. In order to guarantee 10000 mating de-mating cycles for the entire SIROM, further technological fluidic I/F development is needed.

Given the high power involved and the wide operational temperature range, fluid exchange was preferred as heat transportation mean through the interface. System designs considering phase change (heat pipes) were discarded due to the complexity and envelope requirements of the required connectors [7]. A set of technical fluids were considered for heat transportation. For the orbital scenario, glycol/water mixtures, Ammonia or even more elaborated technical fluids such as NOVEC 7500 can be potentially used within the IF. Temperature restrictions are much more severe in the planetary scenario and only a few commercial technical fluids, hydrocarbon based, can be used such as NOVEC 7200 or Dynalene based on their operational temperature range. The thermal standard IF is mainly composed of:

- Two fluid quick connectors (one male and one female) that provide autonomous connectivity between to SIROM IFs. Staubli CGO03 commercial connectors were selected for the demonstration activities in the SIROM project;

- Two flexible lines (metallic bellows), that provide the required motion range for mating and de-mating of the SIROM Ifs;
- A user IF to a SIROM, basically consisting in a 18/" NPT hydraulic elbow fitting;

- Two redundant NTC thermistors to assure temperature is warm enough to not damage the elastomeric seals in the quick fluid connectors during mating process.

Fig. 7 shows the key components of the thermal IF.

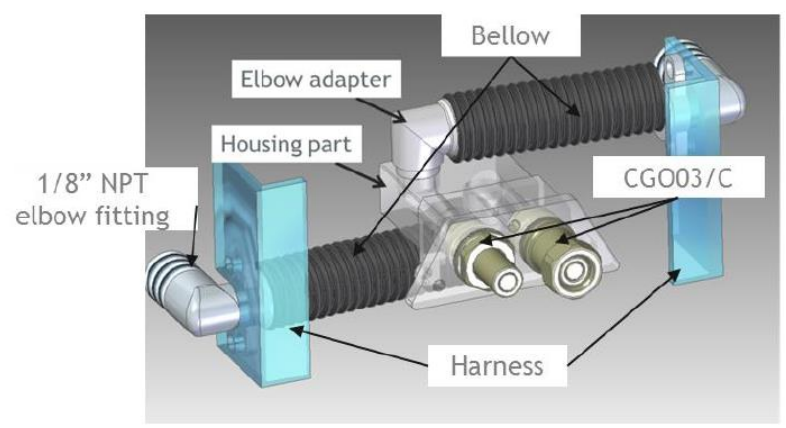

Fig. 7. SIROM thermal interfaces schematics

For SIROM activity, the possibility of implementation of a Close-Loop Heat Exchanger between to APMs equipped with SIROM standard IF is being investigated. These thermal APMs will contain the required components for circulating the fluid between APMs and exchange the heat between a cold sink and a heat source. Two APMs are connected to generate a close-loop heat exchanger. Each of these APMs will mainly contain:

- A compact positive displacement pump;

- A set of check valves to regulate the operation of the APMs;

- A fluid heat exchanger with an standard user IF to the APMs consisting in a $125 \times 90 \mathrm{~mm}$ aluminium plate with 14 M4 threaded holes;

- An expansion vessel to accommodate fluid differential contractions and to accommodate the required extra fluid to compensate fluid leakage after 10000 mating/de-mating operations;

- An Electronic Control Unit (ECU) to manage the operation of the two heat exchange modules.

Fig. 8 shows the hydraulic diagram of one of this APM proposed for verification of the Close-Loop Heat Exchanger concept.

\subsection{Data interface}

The SIROM data interface will support SpaceWire (SpW) interfaces as high-bandwidth data transfer interface and CAN bus interfaces for data transfer. The SpW data interface is composed of 2 links (nominal and redundant) supporting full duplex operation and link rates of up to $100 \mathrm{Mbps}$. The SpW data interface and the electrical link characteristics complies with the European SpaceWire standard ECSS-E-ST-50-12C. 


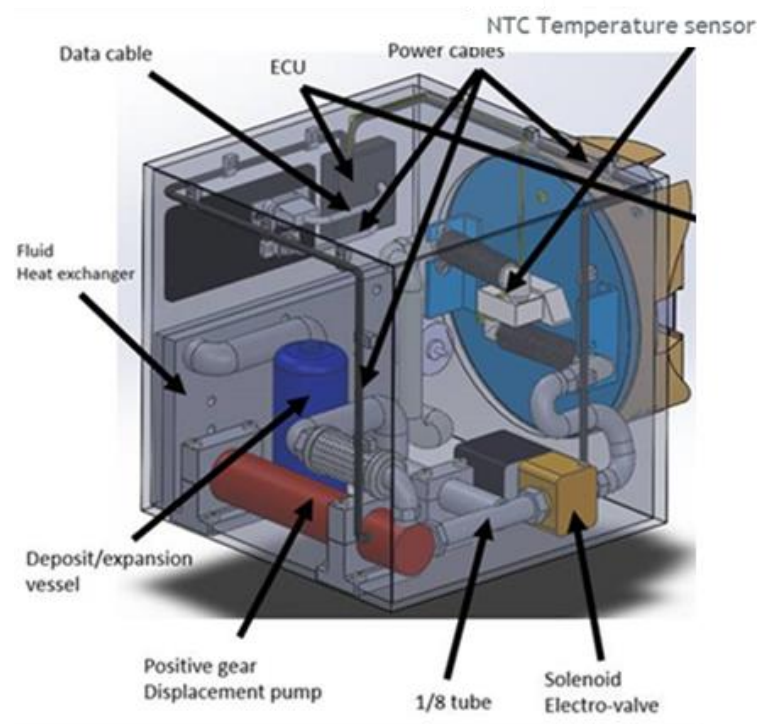

Fig. 8. SIROM with hydraulic diagram linked to an APM

\subsection{SIROM controller}

The SIROM controller is an avionics component part of the SIROM system and connected as a slave device to a dual redundant CAN bus. It receives its commands from a master device remotely connected to the bus (OBC from spacecraft, planetary rover or APM). The controller implements the algorithms that monitor and control:

- the locking and coupling mechanisms of the SIROM;

- the Electrical Interface System (EIS) responsible for switching the $100 \mathrm{~V}$ and $24 \mathrm{~V}$ power lines across the SIROM;

- the dynamic switching of the SIROM controller to the redundant $\mathrm{CAN}$ bus in case of the nominal bus failure in compliance with the ECSS-E-ST50-15C CAN bus extension protocol.

The controller has no control on the data and thermal interfaces as these are implemented as passive connections at the level of the SIROM.

The proposed hardware architecture for the SIROM controller is built on a PCB with a SoC and peripheral components such as memory units, CAN transceivers and a serial interface for debugging purpose. Fig. 9 presents this preliminary architecture. The $\mathrm{SoC}$ is based on a radiation hardened FPGA comprising a number of IP Cores populating a LEON processor, the CAN controllers, an I/O controller for digital and analogue inputs and outputs and memory and DMA controllers to efficiently control the access to all memory units. This SoC qualified for space exploration missions would be suitable for controlling the SIROM as well as for commanding APMs requiring limited computation capabilities.
When deployed as a SIROM controller, the PCB comprises an additional motor controller for the SIROM actuator but has no interface provision for the SpaceWire link as this link is simply implemented as a pass through at the SIROM. When deployed as an APM controller, the $\mathrm{SoC}$ comprises the additional IP Cores of a SpaceWire router that allows the APM controller to switch data between multiple SIROMs.

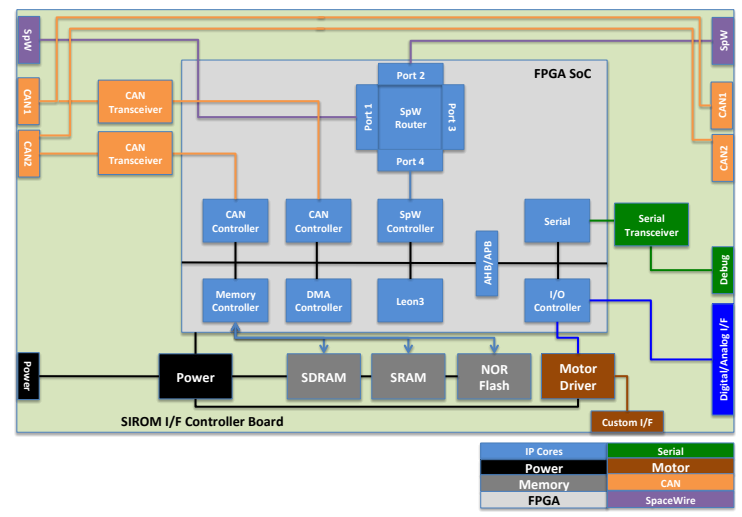

Fig. 9. Preliminary Architecture of a Space Grade Controller for SIROM I/F and APMs

For implementing the SIROM controller, suitable flight proven FPGA technologies exist as e.g. the RTGA, RTAX and RT ProASIC3 manufactured by Microsemi or the Virtex-4 or Virtex-5 manufactured by Xilinx. However, as these components are manufactured in the US and distributed under EAR regulations, the new European FPGA designed by NanoXplore and to be manufactured by STMicroelectronics in 2019 is also considered as an interesting alternative.

- In order to meet the budgetary and programmatic constraints of the SIROM H2020 project, the level of maturity and representativeness imposed on the avionics selected for developing the SIROM controllers is only at TRL4 (breadboard functional validation in laboratory environment). Therefore, readily available low-cost COTS components are used for developing the SIROM controllers but offer the same functional blocks as their space grade counterparts.

- Fig. 10 presents the hardware architecture and COTS components that have been selected for the bread-boarding of the SIROM I/F controllers. 


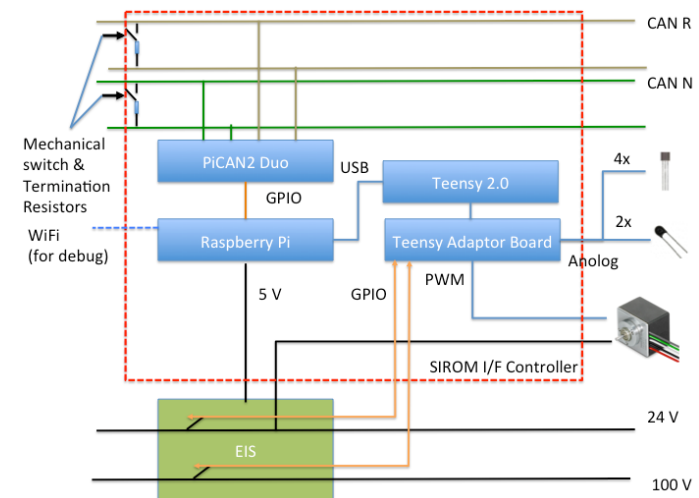

Fig. 10. COTS Breadboard of the SIROM I/F Controller

Because of the low level of integration of the COTS selected for the bread-boarding of the SIROM I/F avionics, there is not enough room to accommodate it inside the SIROM mechanical housing. This avionics is thus housed inside the APM, directly bolted to the structure. The SIROM I/F controller consists of 4 main components:

- Raspberry Pi Zero is to control overall operation of the SIROM interface (communication, control and monitoring).

- $\quad$ Teensy 2.0 , provides additional I/O interfaces (including analogue $\mathrm{I} / \mathrm{O}$ ) and controls $\mathrm{I} / \mathrm{O}$ operations such as reading the sensors and latching switch status, and controlling the motor.

- Teensy 2.0 adaptor board is a simple board consisting of passive components and connectors for interface and conditioning of the actuator/sensors.

- $\quad$ PiCAN2 Duo is to communicate via CAN bus.

The SIROM provides a mechanical switch to properly terminate the CAN bus by a $120 \mathrm{Ohm}$ resistor. When a SIROM is at the end of the bus, the switch is maintained in closed position and this terminates the bus by the resistor. When two SIROMs are connected in the middle of the bus, the SIROM switches are mechanically forced to open disabling the termination resistors. This is shown in Fig.11.
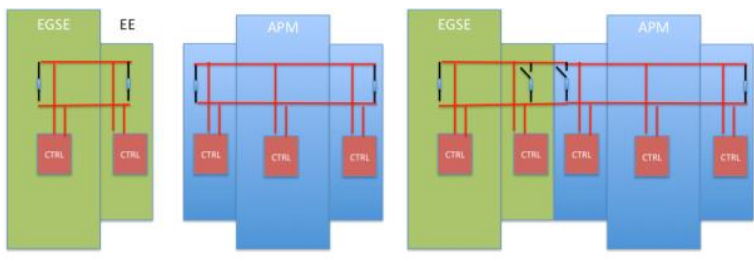
After connection

Fig.11. Termination resistors

\section{Simulations and tests}

The SIROM interface integrates many functionalities, which make this product complex. Therefore, a detailed understanding of how it performs is indispensable to ensure a safe and a reliable operation. The following simulations and tests were designed, based on the life cycle of the interface, using loads from the launch of the SIROM on the launcher to the ones that it could encounter during a planetary or orbital mission. For most of the components that had to be tested, the loads had been isolated and examined separately. In these analyses, thermal effects and structural integrity have been taken into account.

\subsection{Structural analysis}

The global finite element model (FEM) used to test the structural components is shown in Fig. 12, where stress level is analysed and identified for a given load.

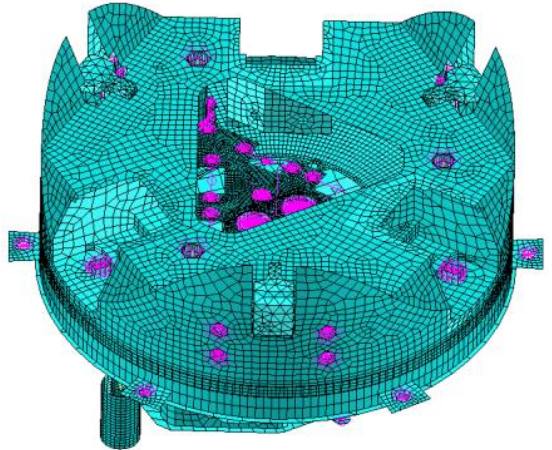

Fig. 12. SIROM FEM model

A summary of the mechanical analysis and calculations is performed to ensure its mechanical load bearing capability through the following:

- Launch load analysis: to prove SIROM is compliant with $15 \mathrm{~g}$ loads;

- Non-nominal load analysis: to evaluate SIROM under $400 \mathrm{~N}$ and $80 \mathrm{Nm}$;

- End-effector loads analysis: to study SIROM under $10 \mathrm{~g}$ sine load up to $100 \mathrm{~Hz}$;

- Gear analysis: to evaluate correct operation according to ISO 6336;

- Latch analysis: to evaluate latches strength and stiffness;

- Capture tab analysis: to evaluate capture tab strength and stiffness;

- Connectors plate analysis: to evaluate strength under the maximum connection forces;

- Spindle/nut analysis: to verify stresses arising from Connectors plate connection.

- Thermoelastic analysis: to study the influence of temperature on latching force and define the clearances required at the different mobile parts to prevent jamming. 
7.2 Thermal analysis

High power thermal interface analysis includes:

- Close-Loop Fluid Heat Exchanger Module flight concept (Pressure Drop Calculations);

- Close-Loop Fluid Heat Exchanger Module demonstration prototype. (Pressure Drop Calculations);

- Close-Loop Fluid Heat Exchanger (FEM Validation).

\subsection{Multi-physics simulations}

As a mechatronic system, SIROM can be considered as a synergistic integrated result of design solutions from several disciplines and requires support from multiperspective of mechatronic design and simulation [8]. Research work has been done in developing multiple level hierarchical modelling in order to achieve multiple perspective, multiple life-phase modelling of a mechatronic system [9] or at macro and micro level modelling to ensure the consistency and traceability of the design process [10]. A multi-perspective simulation is being investigated.

\subsection{Preliminary Test campaign}

\subsubsection{Position-based visual servoing}

The task of visual servoing in robotics consists of controlling a pose (assumed here as position and orientation) of an end-effector of a robotic manipulator to reach a target, based on the visual feedback provided by a camera.

The control can be achieved by either using the Position-Based Visual Servo (PBVS) or Image-Based Visual Servo (IBVS). The PBVS uses a calibrated camera and known features of a target object to determine its pose and thus the relative motion necessary to achieve the final relative pose of the end-effector with respect to (w.r.t.) a target. The IBVS on the other hand uses the image feature extraction directly to derive the required relative motion, without performing pose estimation of the target, [11].

The visual servoing control used in the planetary scenario of the SIROM project is the PBVS and was selected due to its robustness since although the IBVS should be computationally less expensive it presents a challenging control problem due to a highly non-linear relation between the image features and the camera pose [11].

To test the performance and outline the limitations of the developed visual servoing system and its control algorithms three distinct phases were performed and are outlined hereafter:

1. Equipment tests (i.e. development testing at equipment level)

2. Subsystem tests on a testbed (i.e. development testing at subsystem level)

3. Subsystem tests on a robotic platform (i.e. qualification testing at element level)

\subsubsection{Subsystem tests on a testbed}

The scope of subsystem tests on a testbed is to validate new design concepts/techniques at the subsystem level, assess the performance of the visual servoing subsystem in a controlled environment, outline its limitations and provide the input data for the next test phase.

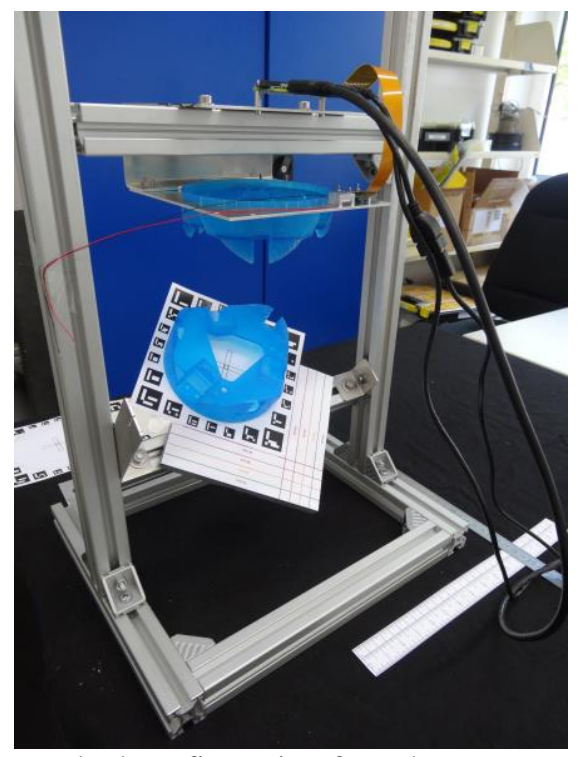

Fig. 13. Testbed configuration for subsystem tests of the visual servoing system (credit: DFKI GmbH 2018)

The configuration of the testbed, illustrated in Fig. 13, consists of a custom-made mounting based on Bosch Rexroth modular profiles, a Raspberry Pi Zero, a raspberry Pi Camera Module v2, a Lumileds LUXEON TX SMD LED, 2 3D printed SIROM interfaces and a target sheet populated with ArUco markers.

The tests on a testbed were performed in two different configurations (i.e. baseline and nominal) under different lighting conditions in order to collect the data regarding the subsystem performance and limitations.

The difference between the baseline and nominal tests consisted of experiments being performed without and with the mounted SIROM interfaces, respectively.

In all of the experiments the relative position and orientation errors of the target w.r.t. the camera was at first considered along different axis separately and then combined. The range of the considered pose error was between 10 and $40 \mathrm{~mm} / \mathrm{deg}$.

The outcome of the tests shows the suitability of the developed visual servoing system to satisfy the requirements of the project and thus the assumed pass criteria which are to provide the target position within \pm $5.5 \mathrm{~mm}$ (i.e. $\pm 5 \mathrm{~mm}+10 \%$ ) and $1.65 \mathrm{deg}$ (i.e. $\pm 1.5 \mathrm{deg}+10 \%$ ).

\subsubsection{Subsystem tests on a robotic platform}

The scope of subsystem tests on a robotic platform was to perform tests of the subsystem on a real world, 
robotic platform in a realistic environment to assess the performance of the visual servoing subsystem under challenging conditions and determine its suitability for the usage in the final OG5 tests.

The tests were performed in the Space Exploration Hall at DFKI GmbH. The rover used in the tests was the SherpaTT (see Fig. 14), a hybrid walking and driving rover with an active suspension system developed for high mobility in irregular terrain [12].

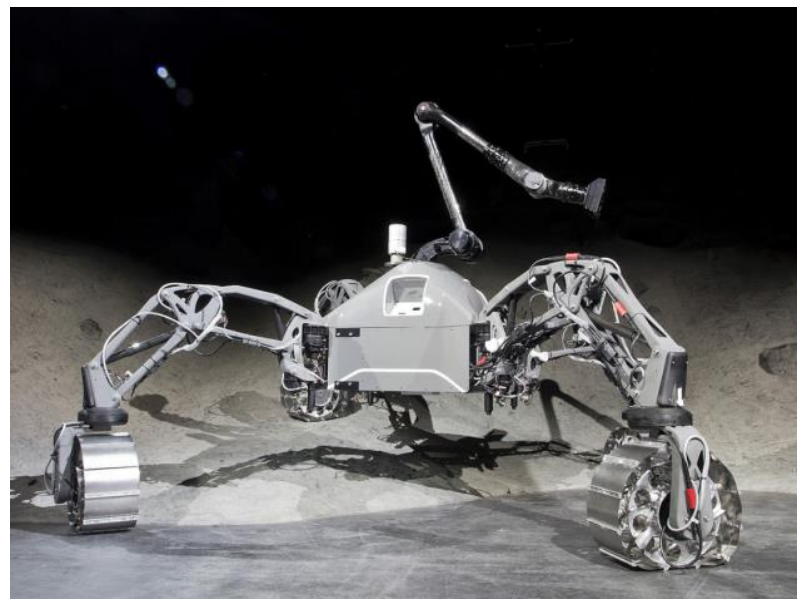

Fig. 14. SherpaTT in the Space Exploration Hall of DFKI (credit: DFKI GmbH 2018)

The rover is also equipped with a manipulator on top of which a mock-up of an auxiliary APM with a mounted camera, for the eye-in-the-hand configuration, was mounted, as visible in Fig. 15. A mock-up of a primary APM was used as a target.

In total, five tests were performed to investigate the ability of interaction between SherpaTT and its manipulator arm with the auxiliary APM and primary APM and assess the performance of the visual servoing subsystem.

The first test contains the transportation of the primary APM by the SherpaTT rover. The primary APM was docked below the rovers body and have been carried at least $10 \mathrm{~m}$ within the surrounding area by different terrain configurations of the Space Hall. As shown in Fig. 15 SherpaTT moves on flat ground as well as on uneven terrain, the wooden obstacle. After reaching of a predefined position within the expoloration area the primary APM safely released on the ground.

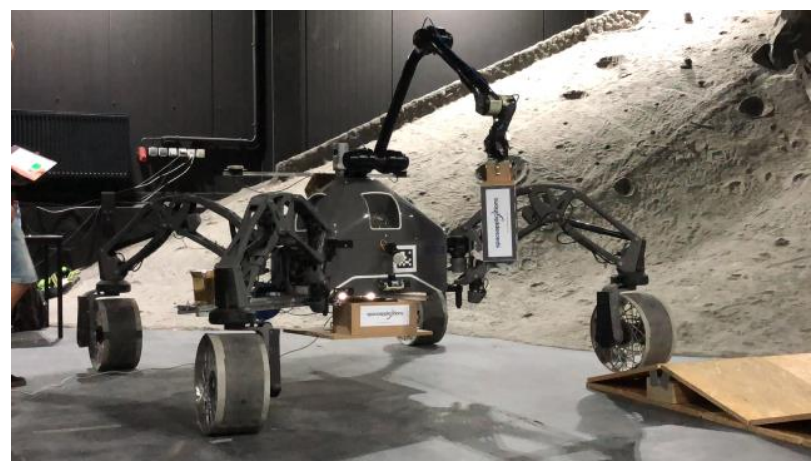

Fig. 15. SherpaTT during its movement on uneven terrain (wooden obstacle) with the primary APM (below) and the auxiliary APM (docked on the manipulator) (credit: DFKI GmbH)

In test 2 the mock up auxiliary APM was manually inserted in the SherpaTT basket and safely transported on different terrain configurations.

In test 3 the auxiliary APM, docked on the manipulator arm of SherpaTT, was inserted by the manipulator arm into the basket and removed from there by a predefined, pre-taught trajectory. Fig. 16 shows the manipulation procedure with the auxiliary APM attached on the manipulator arm of SherpaTT.

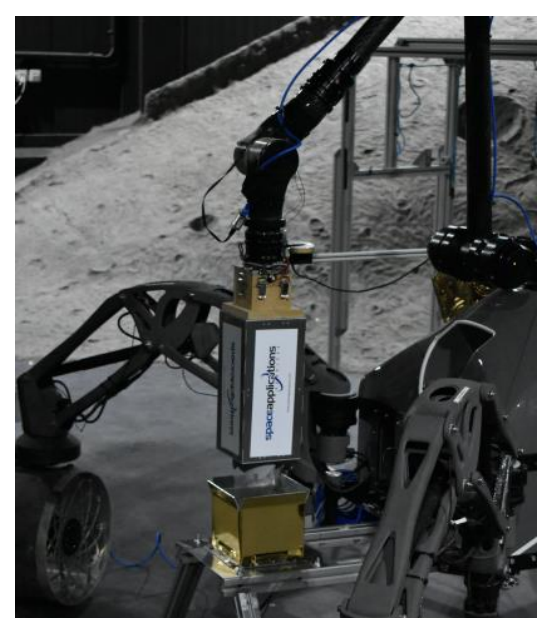

Fig. 16. Auxiliary APM docked on the manipulator arm of SherpaTT during the manipulation procedure to be inserted into the basket (credit: DFKI GmbH)

In test 4 first visual servoing operations were carried out. The camera mounted on the bottom of the auxiliary APM detected the markers on the primary APM. Thereafter, the auxiliary APM was aligned by the manipulator into the docking orientation position and started its docking procedure until $1 \mathrm{~cm}$ from the counterpart interface on the primary APM, see Fig. 17.

The purpose of test 5 was to validate the compliance control of the manipulator arm while manipulating the auxiliary APM within translation and rotation axis. 


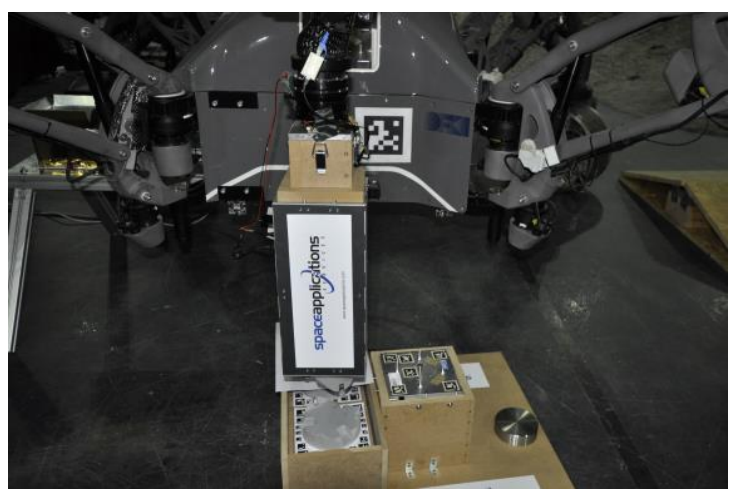

Fig. 17. Docking procedure with visual servoing (credit: DFKI GmbH)

\subsubsection{Thermal test campaign}

A dedicated test campaign for demonstration of the SIROM thermal standard IF and the close-loop heat exchange APMs is foreseen in this activity. Preliminary test of the thermal IF is oriented to characterisation of the requited connection force of the thermal IF.

A dedicated test set up has being manufactured and set up to characterize the required connection force at different temperatures. A stepper motor provides the motion stroke required during mating process and a set of torque and force sensors characterize the required force for connection of the two Thermal IFs. Fig. 18 shows a picture of the test set up.

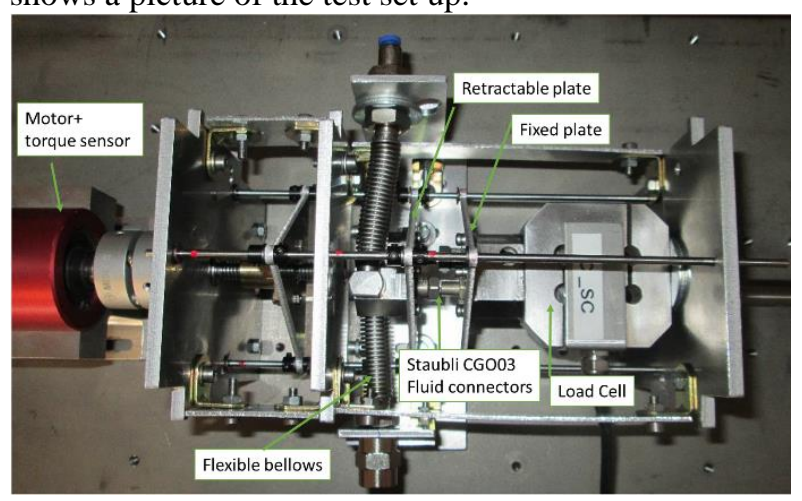

Fig. 18. Test set up for characterization of the connection force

Fig. 19 shows the typical force vs. displacement curve of the Thermal IF in a stroke of $9 \mathrm{~mm}$. Maximum estimated force required for connection of two SIROM thermal IFs at room temperature is $80 \pm 10 \mathrm{~N}$.

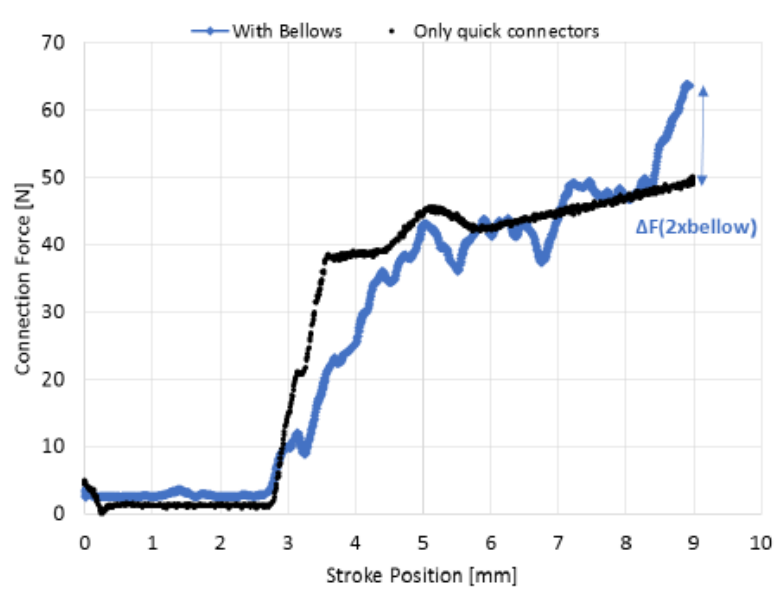

Fig. 19 Force vs. displacement curve of the Thermal IF

\subsection{Performance of the interface}

The performances of SIROM are summarized in Table 1.

Table 1. SIROM performances

\begin{tabular}{|c|c|}
\hline Mass & $<1,5 \mathrm{~kg}$ \\
\hline Dimensions & $\begin{array}{l}128 \mathrm{~mm} \text { diameter } \\
76,6 \mathrm{~mm} \text { height }\end{array}$ \\
\hline Temperature range & $\begin{array}{l}\text { Non-operational: }-128^{\circ} \mathrm{C} \text { to } 50^{\circ} \mathrm{C} \\
\text { Operational: }-110^{\circ} \mathrm{C} \text { to } 50^{\circ} \mathrm{C}\end{array}$ \\
\hline Endurance time & 10000 cycles \\
\hline $\begin{array}{l}\text { Voltage power } \\
\text { lines }\end{array}$ & $\begin{array}{ll} & 100 \mathrm{~V} \\
- & 24 \mathrm{~V} \\
\end{array}$ \\
\hline Electricity transfer & $\begin{array}{l}\text { - } 120 \mathrm{~W} \text { for } 100 \mathrm{~V} \text { line } \\
\text { - } \quad 30 \mathrm{~W} \text { for } 24 \mathrm{~V} \text { line }\end{array}$ \\
\hline Data transfer rate & $\begin{array}{ll}\text { - } & \mathrm{SpW}: 100 \mathrm{Mbit} / \mathrm{s} \\
\text { - } & \mathrm{CAN}: 1 \mathrm{Mbit} / \mathrm{s} \\
\end{array}$ \\
\hline Heat exchange & $2500 \mathrm{~W}$ \\
\hline $\begin{array}{l}\text { Power } \\
\text { consumption until } \\
\text { connection }\end{array}$ & $19 \mathrm{~W}$ \\
\hline Latching force & $1020 \mathrm{~N}$ \\
\hline $\begin{array}{l}\text { Misalignment } \\
\text { tolerance }\end{array}$ & $\begin{array}{ll}- & 10 \mathrm{~mm} \text { axial } \\
\text { - } & 5 \mathrm{~mm} \text { other axes } \\
\text { - } & 1,5^{\circ} \text { all axes } \\
\end{array}$ \\
\hline Latching time & $60 \mathrm{~s}$ \\
\hline Connection time & $102 \mathrm{~s}$ \\
\hline IF to APM & $\begin{array}{l}\text { 6xM3 bolts at } 128 \mathrm{~mm} \text { diameter } \\
\text { circumference }\end{array}$ \\
\hline $\begin{array}{l}\text { Other } \\
\text { performances }\end{array}$ & $\begin{array}{l}\text { Active - Passive SIROM coupling } \\
\text { redundancy } \\
\text { Electric, data and thermal lines } \\
\text { redundancy }\end{array}$ \\
\hline
\end{tabular}

The figures in Fig. 20 show some dimensions of SIROM. Here the micro-D connectors on APM side have been included for a visualization purpose. 

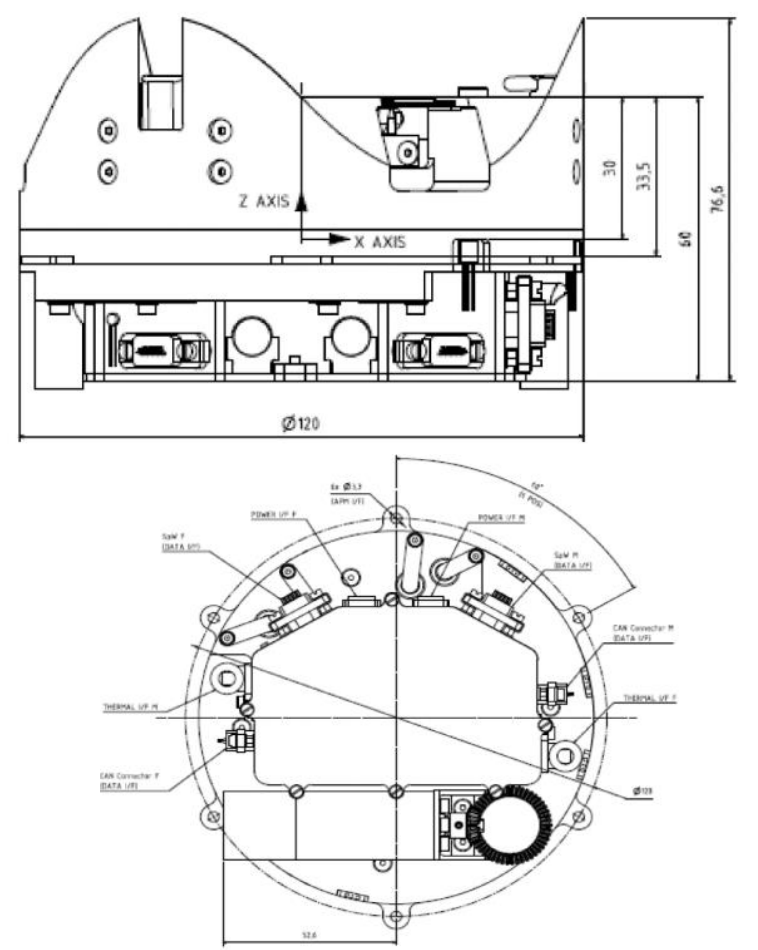

Fig. 20. SIROM main dimensions

Before the integration in SIROM, a number of tests will be performed at individual level on the electrical interface, in order to verify the functional requirements and the performance of the board:

- The capability to manage the required power (120W at $100 \mathrm{Vdc}$ and $30 \mathrm{~W}$ at $24 \mathrm{Vdc}$ ) will be proved in both directions;

- It will be verified the interruption of the lines when the $\mathrm{OFF}$ command $(5 \mathrm{Vdc})$ is given

- It will be verified the continuity of the lines when the $\mathrm{ON}$ command $(5 \mathrm{Vdc})$ is given

- $\quad$ The intervention level of the Under voltage and overcurrent protections will be measured

- Measure of the current vs time when the ON command is given

Once all these tests are passed, the electrical interface will be integrated in the SIROM.

\section{Future applications}

Today's spacecrafts are tailor made very expensive single shot space systems. There is a need to get more benefit from the space systems already launched by extending their lifetime, to overcome the limitations of the launchers set up by the fairing size and to establish satellite platforms which are prepared of being serviced by providing standardized interfaces for APMs. In the last years projects have started e.g. MEV from Orbital ATK or SpaceTug from AIRBUS Defence \& Space which intend to extend the operational lifetime of space system already in space by offering On-Orbit servicing services e.g. refuelling. The target group for these projects are operating satellites which are already in space and not prepared for being serviced. Therefore the services and the business case for these systems are limited. Much bigger business perspective arises if both servicer and client are prepared to service and being serviced respectively.

The space industry will directly benefit from incorporating standardized payload modules with standard interfaces into their spacecraft's. Failed payloads can be replaced rather than launching a new spacecraft. Satellite platforms can be adopted or upgraded to new missions just by adding new subsystems. This would be even more relevant for new satellite constellations e.g. future GPS system, where the performance of the system could be secured, extended or improved by the exchange of valuables equipment e.g. atomic clocks.

New business opportunities will follow. Examples on Earth like the introduction of containers (logistics) or USB drive (IT applications) show the huge potential of standardization.

Utilization of modular building blocks equipped with standardized interfaces offers the opportunity to overcome launcher limitations by assembling on orbit the components launched with successive launches. This leads to space system architectures of large structures which are no longer affected by ground effects and the need of very big launchers.

Manned exploration of Mars, and so called "return to Moon" have recently been publicized and presented as next logical step of space exploration and even human race preservation.

Be as it may, the reality of both close and deep space planetary exploration is almost complete reliance on complex robotic systems designed to travel for long periods of time to far and very hostile environments and perform ever expanding spectrum of scientific and even infrastructural work. Whatever the actual cost of these missions, they are still far cheaper than manned missions, which could aside from the money consume human lives.

This does not mean that there will be no future manned missions, it is actually very easy to imagine planetary robotic systems preparing surface infrastructure on Moon or Mars, before the arrival of the first human crews.

From scientific perspective future robotic systems are going to continue the important search for traces of extraterrestrial life and try to further explain in detail the history of Solar System and even whole universe since its conception.

Last, but not least, is the commercial utilization of robotic systems in what can now be considered emerging space mining industry. At this point space resources utilization seems to be branching into brining valuable resources back to Earth (ore) or to LEO (water) or 
harvesting and utilizing resources in-situ to support future manned expeditions on both Moon and Mars (e.g. water, oxygen, fuel...).

These represent just some of possible future applications where SIROM can and should provide invaluable means of widening operational capabilities of single robotic system, extending power autonomy and even providing means for future upgrades.

The future planetary exploration and commercial missions will take specialized space robotic systems from surfaces of Moon and Mars, to crushing pressures of hot atmosphere of Venus and depths of alien waters of ice covered moons, all the way to mining operations in asteroid belt. While these environments present much more demanding operational requirements than orbital servicing, SIROM has great potential to become Swiss Army Knife type of tool, indispensable in all of future robotic planetary missions, be it scientific exploration, commercial utilization of resources or direct support to manned crews.

\section{Discussions and conclusions}

The space industry faces a lack of confidence in the long term viability of the current business model. The build, launch, operate and replace lifecycle is not a viable concept for the business of future satellite industry. The pressure to replace this concept by a more efficient and sustainable launch and development of a cost saving approach is increasing. The key technologies to develop a sustainable space business are robotics and the standardization of interfaces and payload modules.

Economically, of the highest interest for on orbit servicing is refuelling, upgradability, repair and inspection of space assets. SIROM already supports two of these interests and could be upgraded (the thermal interface already incorporates a fluid loop ) to support a third, namely refuelling by providing also a fluid interface. Therefore SIROM helps to establish satellite servicing applications by supporting the creation of interface standards for satellite servicing

The SIROM development will in the longer term

- Reduce spacecraft cost,

- Extend spacecraft life,

- Reduce the risk of spacecraft failures,

- Improve spacecraft performance and capabilities during their operational time,

- Foster new space business plans and

- Allows faster response to business needs

On orbit assembly will additionally help to overcome the payload size limitations due to launcher limits. This capability offers additional business prospects. With all the advantages modularization and standardization has also its drawbacks. A modular solution is usually not the optimal one in terms of mass or performance for a specific task and there is limited experience in autonomous robotic operations in space. These factors are adding risk and cause higher investments to missions. This leads to some resistance within the industry against the introduction of modular spacecraft and autonomous robotics. The breakeven of modular spacecraft and on orbit servicing will result from longer operational times, enhanced mission flexibility and the distribution of infrastructure investments ( e.g. servicing spacecraft ) over several projects. New business ideas inspired by the new opportunities resulting from this new technologies, lower launch costs and faster excess to space will influence and accelerate the transformation process.

The EU initiative (PERASPERA) helps to reduce technological and business risks by supporting the development of modular interfaces and standardized payload container ( APM ) .

From this initiative, SIROM interfaces have been designed to demonstrate the capability and functionality of a modular standard robotic IF which is needed for future robotic on-orbit servicing, repair and assembly missions. An extensive computer based analyses have been undertaken to ensure its suitability for both orbital servicing mission and planetary exploration requirements. Preliminary tests have also shown its performances meeting the requirements. It is planned for further integrated tests to allow a step-wise on-ground demonstration and validation of the developed and manufactured interfaces.

It is concluded that the SIROM project creates and lays a novel cornerstone for future space robotic missions through the standard robotic interfaces, which will enable a major step forward for On-Orbit Servicing and Assembly (OOS, OOA), realised by the systems to perform much needed robotic operations such as in-situ in-space maintenance, repair and/or assembly to extend life-time of space instructress. Preliminary tests have demonstrated that the design meet the specifications. Specifically, SIROM has been designed to enable the following:

- for orbital operations, to allow direct interaction with cooperative structures for service operations such as maintenance and construction work on technical infrastructure;

- for planetary exploration, robotic systems need to manipulate objects in order to perform scientific investigations and experiments.

The future work would be ideally a demonstration mission of a modular and re-configurable spacecraft. The goal of the mission would feature a small satellite system that, through robotic technology can deploy reconfigure and extendable functionalities. This allows the spacecraft mission to validate the capabilities and the effectiveness of the standard interface SIROM for robotic manipulation of payloads, their exchangeability and a framework for true flexible missions. 
The results of the SIROM development will be used for the design and planning of future European robotic on-orbit servicing and planetary missions planned.

\section{Acknowledgements}

The authors would like to thank all the supporting staff and partners of the SIROM Project: SENER (Spain), Airbus DS Ltd. (UK), Airbus DS GmbH (Germany), Thales Alenia Space S.p.A (Italy), Leonardo S.p.A. (Italy), the University of Strahclyde (UK), German Research Center for Artificial Intelligence (DFKI), Robotics Innovation Center( Germany), TELETEL (Greece), Space Applications Services N.V. (Belgium) and MAG SOAR S.L. (Spain). SIROM is part of the PERASPERA project on Space Robotics Technologies, and has received funding from the European Union's Horizon 2020 research and innovation programme under grant agreement No 730035.

\section{References:}

[1] J. Vinals, E. Urgoiti, G. Guerra, I. Valiente, J. Esnoz, M. Ilzkovitz, D. Cetin, P. Letier, X. Yan, G. Henry, A. Quaranta, W. Brinkmann, M. Jankovic, S.

Bartsch, V. Kollias, N. Pogkas, A. Fumagalli, M. Doermer, Future space missions with reconfigurable modular payload modules and standard interface an overview of the SIROM project, IAC-18-d3.2, 69th International Astronautical Congress, Bremen, Germany, 2018, 1 - 5 October.

[2] Standard Interface for Robotic Manipulation of Payloads in Future Space Missions, project summary http://www.h2020-sirom.eu/, (accessed 12.06.18).

[3] X-T. Yan, W. Brinkmann, R. Palazzetti, C. Melville, Y. Li, S. Bartsch and F. Kirchner (2018) Integrated Mechanical, Thermal, Data, and Power Transfer Interfaces for Future Space Robotics. Front. Robot. AI 5:64. doi: 10.3389/frobt.2018.00064.

[4] R. Palazzetti, K. Donaldson, W. Wenzel, S. Bartsch, $\mathrm{X}-\mathrm{T}$ Yan. Toward a multifunctional interface for future planetary and orbital missions, IAC-18-D3.2, 68th Int. Astronautical Congress, Adelaide, Australia, Sept. 2017.
[5] M. Kortman, S. Ruhl, J. Weise, J. Kreisel, T. Schervan, H. Schmidt, and A. Dafnis, Building block based iBoss approach: fully modular systems with standard interface to enhance future satellites, 66th International Astronautical Congress, Jerusalem, Israel, 2015, 1-11.

[6] M. Jankovic, W. Brinkmann, S. Bartsch, R. Palazzetti, X. Yan, Concepts of active payload modules and end-effectors suitable for Standard Interface for Robotic Manipulation of Payloads in Future Space Missions (SIROM) interface, in: Proceedings of the 2018 IEEE Aerospace Conference, IEEE Aerospace Conference, 2018, Big Sky, Montana, USA, pp.1-16

[7] J.P. Alario and P.J. Otterstedt, “A Heat Pipe Quick Disconnect", 15th Intersociety Conference on Environmental Systems, California, 1985.

[8] Lefèvre J, Charles S, Bosch M, Eynard B, Henner M. Multidisciplinary Simulation of Mechatronic Components in Severe Environments. In: Aiguier M, Bretaudeau F, Krob D, editors. Complex Syst. Des. Manag. Proc. First Int. Conf. Complex Syst. Des. Manag. CSDM 2010, Springer p. 295-304. doi:10.1007/978-3-642-15654-0_21.

[9] P Hehenberger. Perspectives on hierarchical modeling in mechatronic design. Adv Eng Informatics 2014;28:188-97. doi:10.1016/j.aei.2014.06.005.

[10] C. Zheng, P. Hehenberger, J. Le Duigou, M. Bricogne, B. Eynard. Multidisciplinary design methodology for mechatronic systems based on interface model. Res Eng Des 2016. doi:10.1007/s00163-016-0243-2.

[11] P. Corke, Vision-Based Control. In: Robotics, Vision and Control. Springer Tracts in Advanced Robotics, vol 73. Springer, Berlin, Heidelberg, 2011, pp. 455-479

[12] F. Cordes, A. Babu, SherpaTT: A Versatile Hybrid Wheeled-Leg Rover, In Proceedings of the 13th International Symposium on Artificial Intelligence, Robotics and Automation In Space, (iSAIRAS-16), 20.6.-23.6.2016, Beijing, June 2016. 